\title{
The Role of Wireless Communication for Autonomous Military Robot
}

\author{
${ }^{1}$ Sandeep Bhat, ${ }^{2}$ Dr. M. Meenakshi
}

\begin{abstract}
Military applications are very hazardous. Some of those applications are cannot performed by human beings. Hence wireless robots are deployed in such applications. Here for the video transmission from surveillance area to the processing Personal Computer (PC), the FM (Frequency Modulation) is used and also from processing PC to robot commands are transmitted through ASK (Amplitude Shift Keying) modulation.
\end{abstract}

Index Terms -ASK, Autonomous, FM, Modulation, Robot

\section{INTRODUCTION}

A robot is a machine capable of carrying out a difficult operations automatically, especially one programmable by a computer. A robot can be controlled by a human operator, by a long distance. For long distance application wireless communication is used [1]. And risky operations are handled by wireless robots[2].The application areas of mobile robotics in domestic area are cleaning, personal assistance, surveillance, etc., because this area is high in demand[3].Fast standardisation and explosion of wireless robotics increases efficiency, profitability and guarantee sustainability in all application areas of robotics like industrial, defence, scientific, medical and civilian applications[4].

Surveillance robot communicates with device through wireless medium such as Bluetooth. The robot has consisting of some sensors like Humidity, Temperature, Ultra sonic and PIR. Humidity sensor senses the Humidity around the robot. Temperature sensor senses the climate changes, Ultra sonic sensor is used for obstacles presence across the robot path and PIR sensor identifies the human being. And a GSM module receives the sensor information and sends suitable command to the robot for its path planning [5].

The mobile robot can navigate with neighbouring sensor nodes. For this two navigation algorithms are used; first one is used to compute the distance between the mobile robot and each sensor node next the second algorithm computes number of sensor stages [6]. An embedded robotic system based on frequency modulation, to send the commands to remote microcontroller based robots [7].

Organization of this paper is as follows; Section II explains the Block Diagram and its Explanation in detail followed by the Implementation and Results in the section III. Finally the Conclusions are drawn in section IV.

\section{BLOCK DIAGRAM AND ITS EXPLANATION}

The video transmission system based on wireless FM is as depicted in the figure 1 .

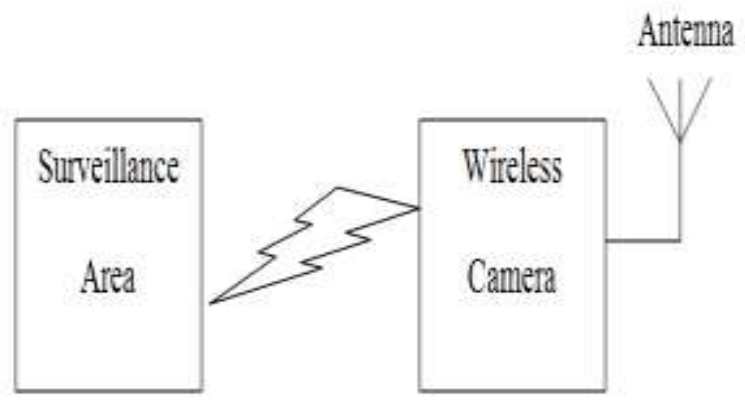

Fig. 1. The transmission system of FM.

The wireless camera captures the image from the military surveillance area as illustrated in figure 1 . The image captured is transmitted towards the receiver through its antenna mounted on it by FM modulation technique. Next at the receiver side, antenna mounted on wireless $2.4 \mathrm{GHz}$ audio /video receives the transmitted signal as shown in the figure 2 . Later it demodulates the FM based video and transmits it to the processing personal computer (PC). The PC sends the appropriate command to the robot via microcontroller 8051 with RF transceiver system. Here RF transceiver system uses ASK modulation and demodulation for data (command) transmission and reception respectively.

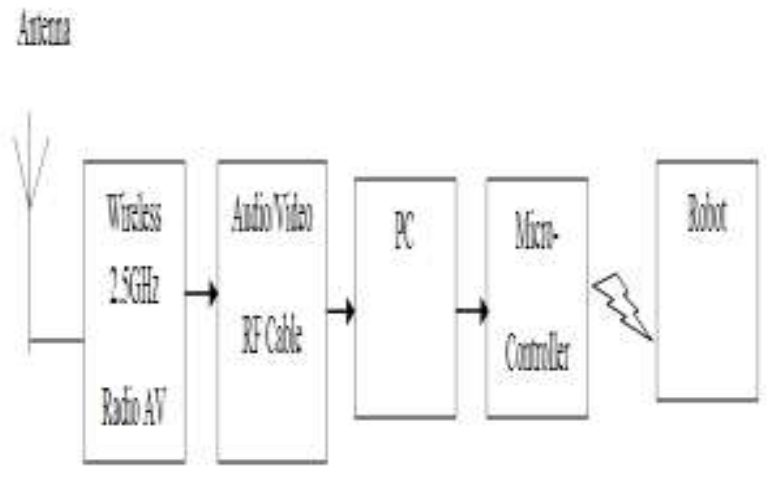

Fig.2. FM Receiver system

\section{IMPLEMENTATION AND RESULTS}

The practical set-up of the FM based wireless video transmission system is as illustrated in the figure 3 . This

\footnotetext{
Manuscript received on November 15, 2016.

${ }^{1}$ Associate Professor, E\&CE Dept. S. I.T.,Mangaluru, INDIA

${ }^{2}$ Professor \& Head, Dept. I.T. Dr. AIT, Bengaluru, INDIA.
} 
figure includes the battery powered wireless camera as the transmission part as given in figure 3a. And $\mathrm{RF}$ radio $\mathrm{A} / \mathrm{V}$ part is incorporated in the receiver part as depicted in figure $3 \mathrm{~b}$ and the RF receiver connected video dongle to $\mathrm{PC}$ is as shown in the figure $3 \mathrm{c}$. Here the video transmission between the transmitter and receiver part are taking place through FM modulation/demodulation technique till the distance of 9 meters.

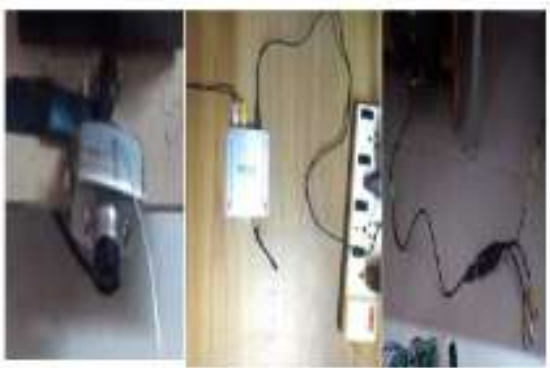

a b c

Fig.3. Wireless Video Transmission/Reception System

The information which is to be transmitted is as given in the figure $4 \mathrm{a}$. After frequency modulation of the message signal as shown in figure $4 \mathrm{a}$ is as depicted in figure $4 \mathrm{~b}$.

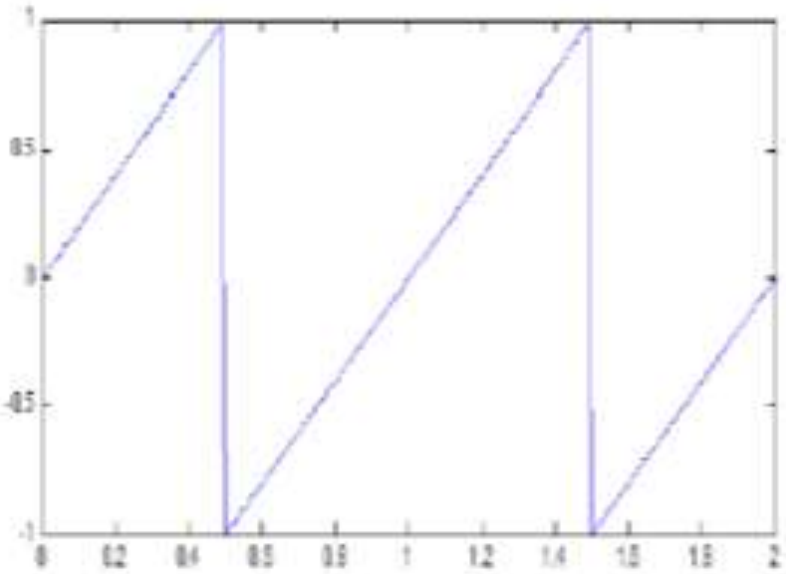

Fig. 4a: A message signal

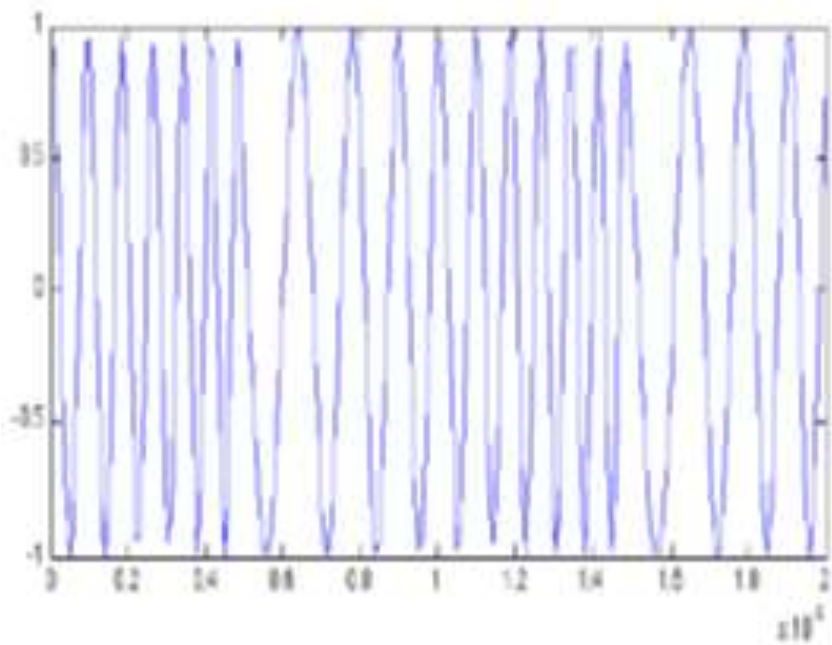

Fig. 4b. FM Demodulated signal. (Courtesy: www.mathworks.com)

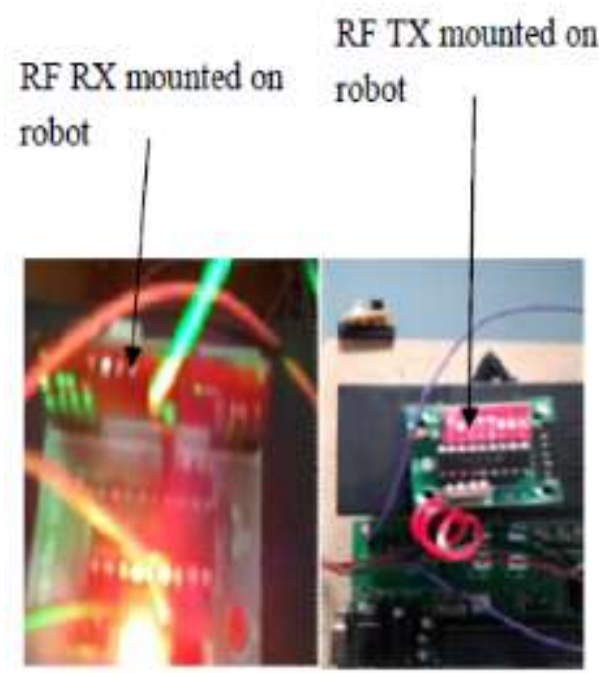

Fig.5. RF transmitter and receiver module.

The transmitter and receiver section of the $\mathrm{RF}$ communication robot for the long distance are as given in the figure 5. Here the RF receiver and transmitter sections are mounted on the transmitter and receiver section of the military robots to perform the given task. The command is transmitted to the robot for its path planning by the ASK modulation and is demodulated by ASK demodulation technique as shown in the figure 6 , to control the robot's motion.
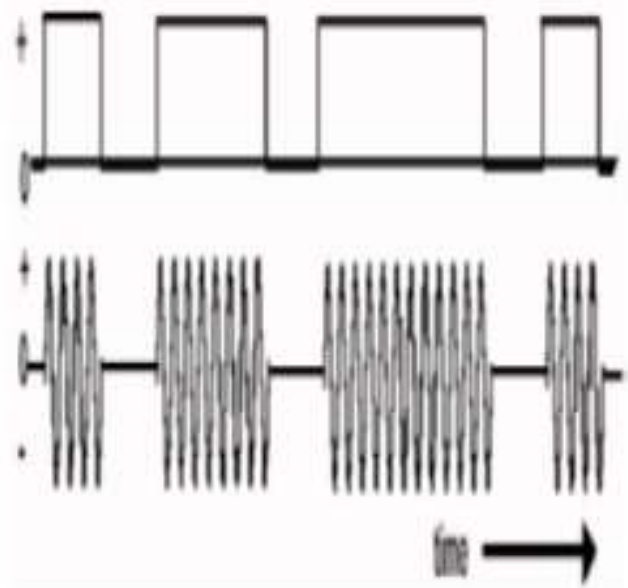

Fig.6. ASK Waveforms. (Courtesy: www.srmuniv.ac.in)

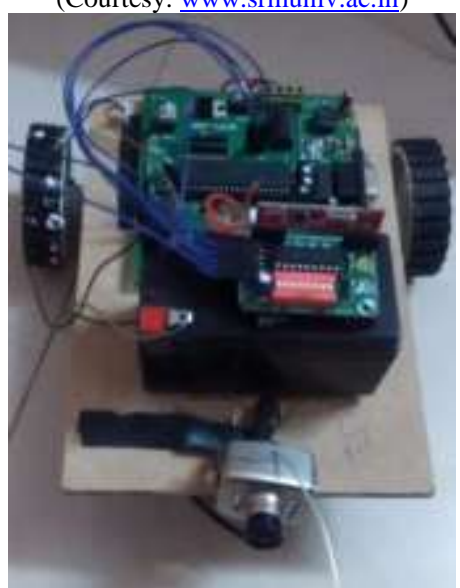

Fig.7. Robot Prototype. 
The prototype of the robot with wireless camera and RF receiver is as illustrated in the figure 7.

\section{CONCLUSION}

This paper gave solutions to solve very hazardous military applications are performed by wireless robots. These applications are performed by processing $\mathrm{PC}$ by receiving video of the surveillance area with the help of FM modulation/demodulation process. Here after processing the video to identify the obstacle along the path of the robot, the microcontroller interfaced with PC sends the appropriate command to autonomous robot by ASK modulation technique along with RF modules. Hence optimized path of the robot is determined.

\section{REFERENCES}

[1] Kunal, Rohan Gaikwad and Ajaysingh Rajput, "Wireless Controlled Surveillance Robot" , International Journal of Advance Research in Computer Science and Management Studies,Volume 2, Issue 2, 2014, pp. 436-441.

[2] Sivabalan Arumugam,Ritesh Kumar Kalle and Anand R. Prasad, "Wireless Robotics: Opportunities and Challenges", Wireless Personal Communications (Springer), June 2013, Volume 70, Issue 3, pp. 10331058.

https://doi.org/10.1007/s11277-013-1102-3

[3] Henrik Schiøler, Thomas Skjødeberg Toftegaard, "Wireless Communication in Mobile Robotics a Case for Standardization", Wireless Personal Communications (Springer), June 2012, Volume 64, Issue 3, pp. 583-596. https://doi.org/10.1007/s11277-012-0602-x

[4] R. Prasad - M. Ruggieri, "“Wireless Robotics: Research and Standardization", published by Springer Science+Business Media New York, 2013, Volume 70, Issue 3, pp 1029-1032.

[5] M. Balakrishnan; S. Gowthaman ; S.P. Jaya Kumaran ; G. Rathana Sabhapathy, "A smart spy robot charged and controlled by wireless systems", proceeding of International Conference on Innovations in Information, Embedded and Communication Systems (ICIIECS), 2015. https://doi.org/10.1109/iciiecs.2015.7193096

[6] Woo-Yong Lee,Kyeong Hur, Kwang-il Hwang, Doo-Seop Eom and Jong-Ok Kim, "Mobile Robot Navigation Using Wireless Sensor Networks without Localization Procedure" , Wireless Personal Communications(Springer),2012, Volume 62, Issue 2, pp. 257-275. https://doi.org/10.1007/s11277-010-0052-2

[7] Changjiu Pu, "A Robotic System Based on Wireless Control" ,Information Computing and Applications, book chapter of the series Communications in Computer and Information Science, Volume 307,2012 ,pp. 500-506.

https://doi.org/10.1007/978-3-642-34038-3_69

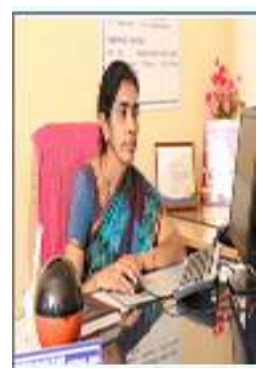

Dr. M Meenakshi obtained B.E. in Instrumentation Technology from SJCE, Mysuru and M.Tech in Instrumentation and Control from IIT Madras and $\mathrm{Ph} \mathrm{D}$ in Aerospace Engineering from IISc, Bengaluru, India.

Author is currently working as Professor \&Head, Dept. I.T. Dr. AIT, Bengaluru, INDIA. Author is having thirty years of teaching and ten years of research experience. Published more than 50 research papers in national/international conferences and journals.

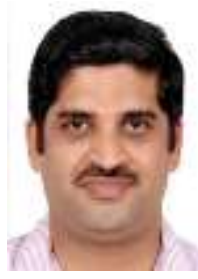

and journals.
Mr. Sandeep Bhat obtained B.E. in Electronics and Communication and M.Tech in Digital Electronics and Communication from VTU, Belagavi, INDIA. Presently persuing $\mathrm{Ph} \mathrm{D}$ in the specialization Autonomous Robot.

Author is currently working as associate professor ECE department, Srinivas Institute of Technology, Mangaluru, India. Author is having thirteen years of teaching and five years of research experience. Published 24 research papers in national/international conferences 